\title{
PHOTOLUMINESCENCE KINETICS OF YAG CRYSTALS ACTIVATED WITH Ce, AND Ce AND Mg*
}

\author{
J. Barzowska, A. Kubicki, M. Grinberg
}

Institute of Experimental Physics, Gdańsk University, 80-952 Gdańsk, Poland

S. KACZMAREK

Institute of Optoelectronics, Military University of Technology, 01-489 Warsaw Poland

\section{Z. ŁUCZYŃSKI}

Institute of Electronic Materials Technology, 01-919 Warsaw, Poland

\author{
A.J. Wojtowicz and Cz. Koepke
}

Institute of Physics, N. Copernicus University, 87-100 Torun, Poland

(Received September 11, 1998; in final form January 29, 1999)

A basic spectroscopic measurements of luminescence, absorption, luminescence excitation spectra and emission kinetic measurements on YAG crystals activated with cerium and magnesium are presented. We report that the $\mathrm{Ce}^{3+}$ luminescence decay constant, at $65 \mathrm{~ns}$, is independent of Ce concentrations (from 0.05 to $0.2 \%$ ) and that it does not change with the presence or absence of the $\mathrm{Mg}$ co-dopant. Nevertheless, we find that under pulsed laser excitation at $290 \mathrm{~nm}$ the rise time in Ce luminescence time profiles is effectively shorter in the $\mathrm{Mg}$ co-doped samples.

PACS numbers: 71.55.-i, 78.47.+p, 78.55.-m

\section{Introduction}

An electronic structure of octahedrally coordinated $\mathrm{Ce}^{3+}$ ion is determined by the single electron $4 f$ and $5 d$ electronic configurations of the central ion whose energies are modified by local crystal field. In the case of $\mathrm{Y}_{3} \mathrm{Al}_{5} \mathrm{O}_{12}$ (YAG), where the $\mathrm{Ce}^{3+}$ ions occupy the $\mathrm{Y}^{3+}$ sites of $D_{2}$ symmetry the covalency effect reduces the $4 f-5 d$ separation energy to $35000 \mathrm{~cm}^{-1}$. The splitting of the $d$ fivefold degenerated state results in the structured absorption [1] with well-separated peaks at

*The results of this paper were initially presented at The Jablonski Centennial Conference on Luminescence and Photophysics, July 23-27, 1998, Torun', Poland. 
$450 \mathrm{~nm}, 340 \mathrm{~nm}, 270 \mathrm{~nm}$ and $225 \mathrm{~nm}$, related to spin allowed transitions originated from the ${ }^{2} F_{5 / 2}$ ground state. The broad band (also parity allowed) transitions from the lowest $d$ term to the ${ }^{2} F_{7 / 2}-{ }^{2} F_{5 / 2}$ splitted ground electronic manifold results in the green yellow double band emission. This emission was considered as very promising for obtaining tunable laser action and although it has been shown that a large excited state absorption usually prevents lasing in $\mathrm{YAG}: \mathrm{Ce}^{3+}$ [2], the material is still very interesting as a possible scintillator or X-ray phosphor.

The final step of the scintillation process is the radiative recombination of thermalized electron-hole pairs on $\mathrm{Ce}^{3+}$ ions. Therefore, the co-doping of the scintillating material with dopants that can effectively control free carrier lifetimes and concentration may improve the performance of the material on one hand and, on the other, can shed some light into processes of free carrier capture and nonradiative recombination.

It is expected that the $\mathrm{Mg}^{2+}$ ion introduces the deep acceptor level in the vicinity of the valence band of YAG and, when occupied by electron, forms a negatively charged center, characterised by a large cross section for the hole capture. Magnesium can also possibly contribute to the absorption, as observed, via the acceptor level - conduction band transitions. There is, however, no visible luminescence related to magnesium.

\section{Experimental results}

We have at our disposal single crystals of: YAG:Ce (with weight concentrations of $\mathrm{Ce}-0.05,0.1$ and $0.2 \%)$ and $\mathrm{YAG}: \mathrm{Ce}, \mathrm{Mg}(0.2 \% \mathrm{Ce}$ and $1 \% \mathrm{Mg})$ grown by the Czochralski technique at the Institute of Electronic Materials Technology in Warsaw. The absorption spectra are presented in Fig. 1. For all concentrations one can see characteristic $\mathrm{Ce}^{3+}$ absorption bands peaking at 210, 225, 270, 340, and $450 \mathrm{~nm}$, and the absorption edge at $190 \mathrm{~nm}$ related to the band to band transition in YAG. For the sample co-doped with $\mathrm{Mg}$ there is clearly an additional absorption that effectively lowers the absorption edge to about $300 \mathrm{~nm}$. We propose that this absorption is caused by transitions between the acceptor state and the conduction band. By comparing the absorption edges of YAG:Ce, $\mathrm{Mg}$ and YAG:Ce (or undoped) we estimate the energy of the hypothetical $\mathrm{Mg}^{2+}$ acceptor state to be about $2 \mathrm{eV}$ above the valence band.

In Fig. 2 the $\mathrm{Ce}^{3+}$ luminescence excitation spectra are presented. Clearly the excitation into the $\mathrm{Mg}^{2+}$-related absorption band does not produce any $\mathrm{Ce}$ luminescence. Only the characteristic intracenter transitions of $\mathrm{Ce}^{3+}$ ion can be identified in the $\mathrm{Ce}$ excitation spectrum of the material co-doped with magnesium (curve 3). Moreover, for YAG:Ce, $\mathrm{Mg}$, the excitations with wavelengths shorter than $300 \mathrm{~nm}$ (with an energy above the absorption edge related to $\mathrm{Mg}$ ) result in distinctly weaker Ce luminescence than in the case of YAG doped only with Ce of the same concentration. It is particularly important in the spectral region between 250 and $300 \mathrm{~nm}$, where the absorption spectrum related to $\mathrm{Ce}^{3+}$ is rather weak. The effect can be simply explained by the fact that the strong $\mathrm{Mg}$ absorption in that region effectively lowers the number of photons that can be absorbed by $\mathrm{Ce}$. The second contribution, coming from the competition between $\mathrm{Ce}$ and $\mathrm{Mg}$ ions working as recombination centers, will be discussed below in more detail. 

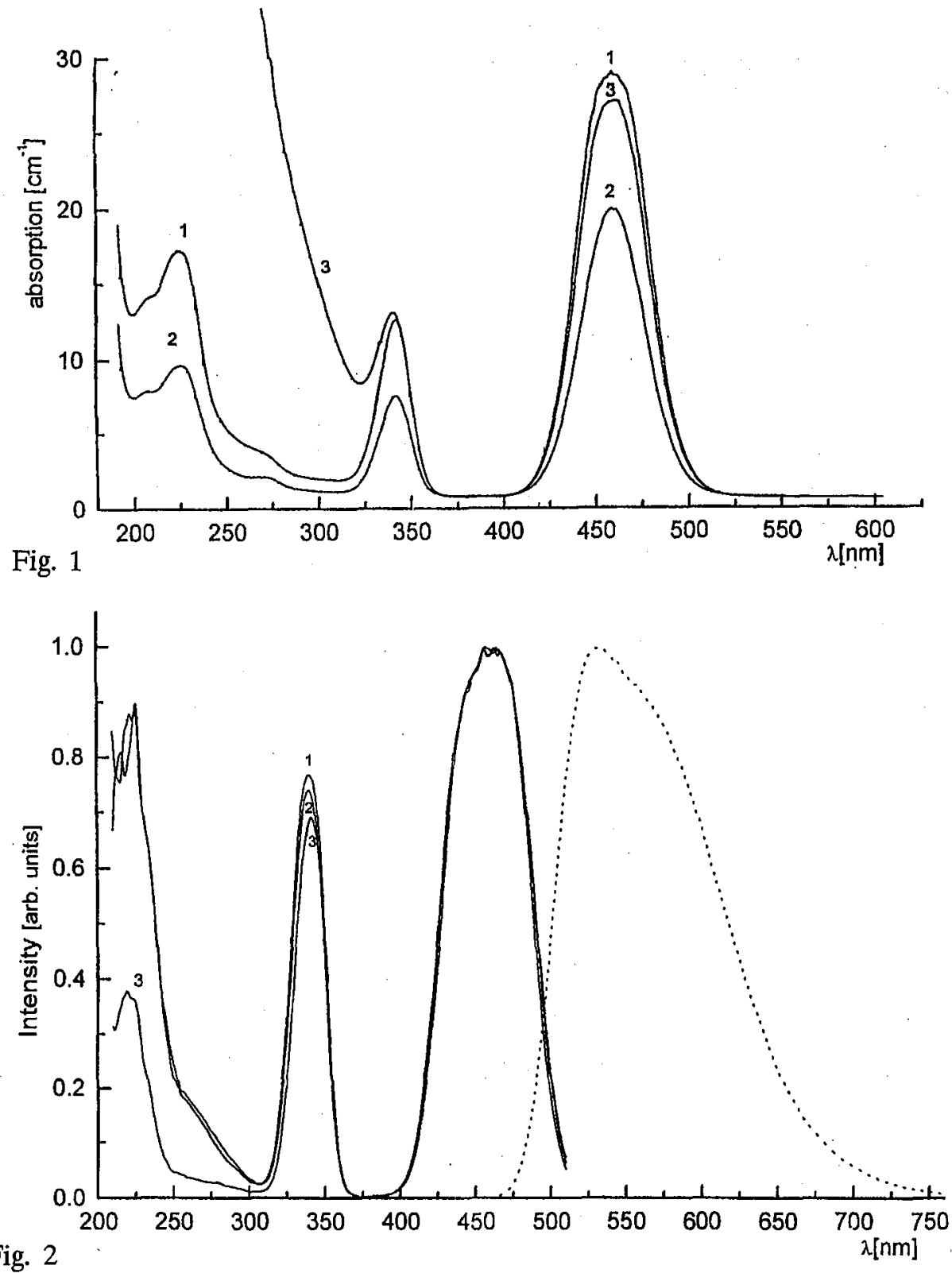

Fig. 1. Absorption spectra of YAG doped with Ce and Mg. Curves 1 and 2 correspond to YAG:Ce with Ce concentration $0.2 \%$ and $0.1 \%$, respectively, curve 3 corresponds to YAG: $\mathrm{Ce}, \mathrm{Mg}$ with concentrations $0.2 \% \mathrm{Ce}$ and $0.1 \% \mathrm{Mg}$.

Fig. 2. Luminescence excitation spectra (luminescence was monitored at $550 \mathrm{~nm}$ ). Curves 1 and 2 correspond to YAG:Ce with Ce concentration $0.2 \%$ and $0.1 \%$, respectively, curve 3 corresponds to YAG:Ce, $\mathrm{Mg}$ with concentrations $0.2 \% \mathrm{Ce}$ and $0.1 \% \mathrm{Mg}$. Dashed curve corresponds to the luminescence spectrum. 
For luminescence kinetic measurements we have excited crystals by using a system consisting of YAG:Nd and dye lasers, both with frequency doubling, working in a mode-locking regime and providing laser pulses of only a few tens of picoseconds at the wavelength of $290 \mathrm{~nm}$. Thus, we excite the $\mathrm{Ce}^{3+}$ above the second excited $5 d$ state. However, since the ground state absorption of $\mathrm{Ce}^{3+}$ at this particular wavelength is very weak, we have to consider, in addition, the excitation by creation of the electron-hole pairs via a two-photon process, or creation the electron-hole pairs by two-electron excitation (simultaneous transitions; from valence band to acceptor state and from acceptor state to conduction band). An important consideration is that if the latter two-particle excitation processes take place then the unavoidable steps including the thermalization of the electrons and holes and the energy transfer to the $\mathrm{Ce}^{3+}$ ions must necessarily delay the Ce luminescence. The emission signal has been detected with a single photon counting technique using a microchannel plate detector. In all cases we have obtained strongly single exponential luminescence decays. It concerns the samples doped with cerium as well as the samples doped with cerium and magnesium. The obtained decay constant being equal to $65 \mathrm{~ns}$ is characteristic of the $\mathrm{Ce}^{3+}$ luminescence $[3,4]$. The measured decays are presented in Fig. 3a.
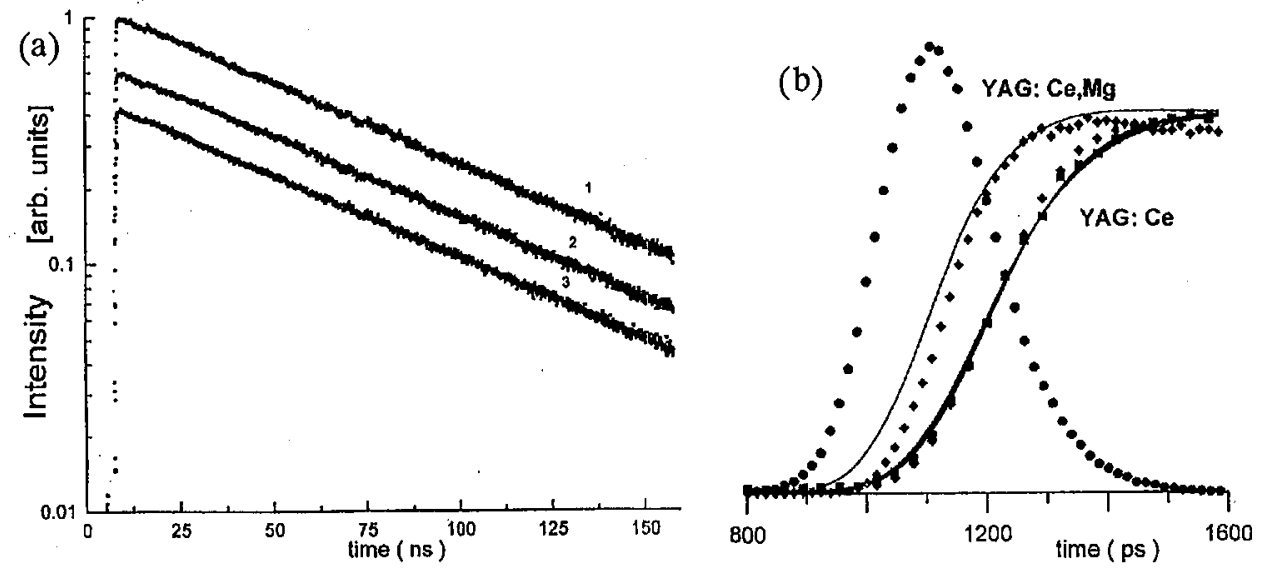

Fig. 3. Luminescence profiles. (a) $\mathrm{Ce}^{3+}$ luminescence decay, decays 1 and 2 correspond to YAG:Ce with Ce concentration $0.1 \%$ and $0.2 \%$, respectively, decay 3 corresponds to YAG:Ce, $\mathrm{Mg}$ with concentrations $0.2 \% \mathrm{Ce}$ and $0.1 \% \mathrm{Mg}$. (b) $\mathrm{Ce}^{3+}$ luminescence rising. Black circles correspond to the response of the instrumental profile. The experimental luminescence intensity profiles are indicated by squares. The solid curves represent theoretically reproduced profiles (detailed descriptions in the text).

It is interesting to note that rising parts of luminescence time profiles in Ce-doped and Ce-doped, Mg-codoped crystals differ quantitatively. In Fig. $3 \mathrm{~b}$ the time dependence of the cerium luminescence intensity on a picosecond scale is presented. In this figure we present only the rising part of the measured time profile. One can see that the luminescence signal is shifted in time with respect to the excitation pulse. This shift is independent of concentration and is the same for 
materials with 0.1 and $0.2 \%$ of Ce. On the other hand we note the large decrease in this shift for the emission from the sample co-doped with $\mathrm{Mg}$.

\section{Deexcitation kinetics}

For the quantitative analysis of measured luminescence time profiles we must take into account the fact that the experimental profile $I_{\mathrm{e}}(t)$ is represented by the folding integral

$$
I_{\mathrm{e}}(t)=\int \mathrm{d} t^{\prime} F\left(t-t^{\prime}\right) I\left(t^{\prime}\right)
$$

of the true intensity time profile $I(t)$ and the instrumental profile $F(t)$.

The instrumental profile has been measured and is given in the numerical form as represented by dots in Fig. 3b.

To get the true luminescence time profile, $I(t)$, we have to consider the time evolution of the excited system. We assume that at the beginning the system consists of unexcited $\mathrm{Ce}^{3+}$ ions and free unthermalized electrons and holes. First, electrons and holes relax step by step with the emission of phonons and, after the electron-hole pair energy is transferred to the Ce ion, there is the relaxation within the ion itself. Eventually, the process is completed by radiative transitions between the metastable excited state and the ground state, which are the lowest $d$ and $f$ states of $\mathrm{Ce}^{3+}$ ion, respectively.

In the general approach one considers a system consisting of $k$ excited states characterised by some specific nonradiative decay constants, $\tau_{\mathrm{nr}}(k)$, and the $k+1$ state that plays a role of a metastable final state (from this state the only transition allowed is the radiative one).

The kinetics of the system is described by the following set of equations:

$$
\begin{aligned}
& \frac{\mathrm{d} N_{1}}{\mathrm{~d} t}=\frac{-N_{1}}{\tau_{\mathrm{nr}}(1)}, \\
& \frac{\mathrm{d} N_{k}}{\mathrm{~d} t}=\frac{N_{k-1}}{\tau_{\mathrm{nr}}(k-1)}-\frac{N_{k}}{\tau_{\mathrm{nr}}(k)}, \\
& \frac{\mathrm{d} N_{k+1}}{\mathrm{~d} t}=\frac{N_{k}}{\tau_{\mathrm{nr}}(k)}-\frac{N_{k+1}}{\tau_{\mathrm{r}}},
\end{aligned}
$$

where $N_{i}$ is the occupation number of $i$-th state and $\tau_{\mathbf{r}}$ is the radiative decay time. Equations (2) can be solved numerically. For initial conditions: $N_{1}(0)=N_{1}^{0}$ and $N_{k}(0)=0$, for any $k \neq 1$, and assuming that each intermediate state is characterised by the same nonradiative decay constant $\tau_{\mathrm{nr}}$ one obtains the analytical formula describing the time dependence of the metastable state occupation number

$$
\begin{aligned}
& N_{k+1}(t)=\frac{(-1)^{k} N_{1}^{0}}{\left(1 / \tau_{\mathrm{r}}-1 / \tau_{\mathrm{n}}\right)^{k} \tau_{\mathrm{nr}}(k)} \exp \left(-t / \tau_{\mathrm{r}}\right) \\
& \quad+\frac{N_{1}^{0}}{\tau_{\mathrm{nr}}(k)} \frac{1}{(k-1) !} \exp \left(-t / \tau_{\mathrm{nr}}\right) \sum_{i=0}^{k-1}(-1)^{i} \frac{(k-1) ! t^{k-1-i}}{(k-1-i) !\left(1 / \tau_{\mathrm{r}}-1 / \tau_{\mathrm{nr}}\right)^{i+1}}
\end{aligned}
$$

In the simplest case of the two excited states one obtains

$$
N_{2}(t)=N_{1}^{0} \exp \left(-t / \tau_{\mathrm{r}}\right)-N_{1}^{0} \exp \left(-t / \tau_{\mathrm{nr}}\right) .
$$


Good approximation of our system is the three-state model, where $N_{1}, N_{2}$, and $N_{3}$ are the occupation numbers of the hot electron-hole pairs, thermalized electron-hole pairs and $\mathrm{Ce}^{3+}$ in the first excited metastable state, respectively. For this model assuming that $\tau_{\mathrm{nr}} \ll \tau_{\mathrm{r}}$, we obtain

$$
N_{3}(t)=N_{1}^{0} \exp \left(-t / \tau_{\mathrm{r}}\right)-N_{1}^{0}\left(\frac{t}{\tau_{\mathrm{nr}}}+1\right) \exp \left(-t / \tau_{\mathrm{nr}}\right) .
$$

The three-excited-state model can be easily generalised to include the case of different nonradiative decay rates in different states. For this case we obtain

$$
N_{3}(t)=N_{1}^{0} \exp \left(-t / \tau_{\mathrm{r}}\right)-\frac{N_{1}^{0}}{\tau_{2}-\tau_{1}}\left[\tau_{2} \exp \left(-\mathrm{t} / \tau_{2}\right)-\tau_{1} \exp \left(-\mathrm{t} / \tau_{1}\right)\right]
$$

In relation (6) we omitted the subscript " $\mathrm{n}$ " at nonradiative decay time. The first component in formulas (4), (5), and (6) describes the decay, whereas the second one corresponds to the rise in the luminescence time profile. In our calculations we have used the three-excited-state model for which the emission intensity is represented by Eq. (6).

To estimate the respective nonradiative decay time constants we calculated the luminescence time profile according to Eq. (1), using relation (6) for modeling the true intensity profile. We found that for YAG:Ce, independently of cerium concentration, the global relaxation time is of the order of 100 ps. In Fig. 3b the different solid curves represent the calculated luminescence rises for different pairs of $\tau_{1}$ and $\tau_{2}$ whose sum is always $100 \mathrm{ps}$ ( $\tau_{1}$ was changed between 0 and $50 \mathrm{ps}$ ).

The best fit to the experimental data for YAG:Ce, $\mathrm{Mg}$ has been obtained under assumption that relaxation of the system to the metastable state is immediate, $\tau_{1}+\tau_{2}=0$, (see the respective solid curve in Fig. $3 \mathrm{~b}$ ). It is possible that the effect of the diminishing of the luminescence rise time is caused by the very fast capturing of free holes by the negatively charged $\mathrm{Mg}^{2+}$ ions. As the result the free holes become unavailable to $\mathrm{Ce}$ ions. This process significantly shortens the $\mathrm{Ce}^{3+}$ luminescence rise time as well as diminishes the emission intensity. This is

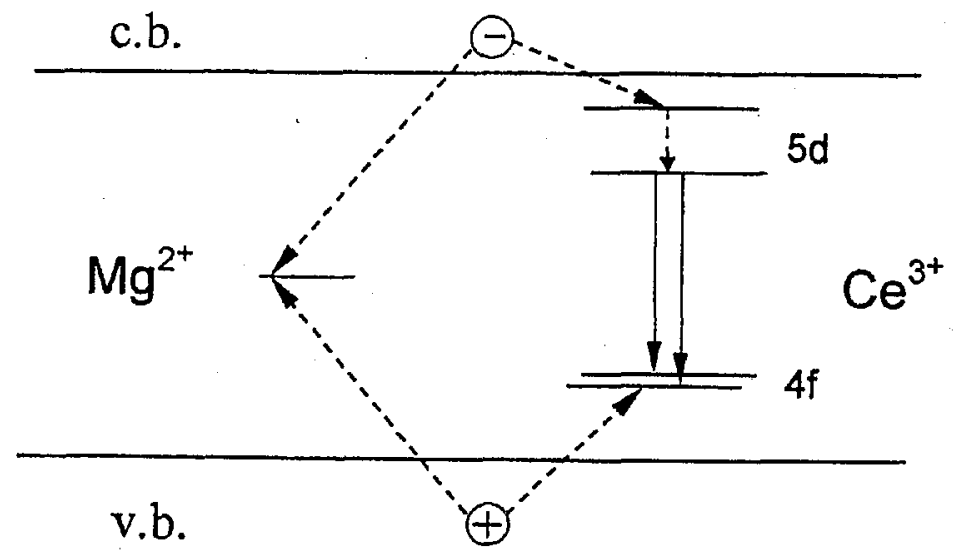

Fig. 4. Diagram representing electron-hole relaxation in the YAG:Ce,Mg system. Solid and dashed arrows correspond to the radiative and nonradiative transitions. 
seen when we excite YAG:Ce, $\mathrm{Mg}$ with the photons of the wavelength shorter than $300 \mathrm{~nm}$. The effect of hole capturing by $\mathrm{Mg}$ is observed also by monitoring the $\mathrm{Ce}^{3+}$ thermoluminescence, which is much weaker in the case of samples co-doped with magnesium [5]. The processes described above are presented in Fig. 4.

\section{Conclusions}

One has to discuss the validity of our kinetic model. Actually, we do not have sufficiently sensitive experimental tools to analyse the details of the deexcitation processes, especially to quantify the nature of all intermediate excited states. Nevertheless, we are able to describe the luminescence kinetics in YAG:Ce and YAG:Ce, $\mathrm{Mg}$ systems using a simple three-excited-state model that explains the experimental observations reasonably well. At the beginning the excited state of the system is that of the electron-hole pair created by a two-particle process. Then the carriers thermalize almost immediately (on a time scale available to us). It seems further that the thermalized carriers need a period of time of about 100 ps to be captured at $\mathrm{Ce}^{3+}$ ion. The final non-radiative process is the intraconfigurational relaxation of the excited cerium ions, which also seems to be fast. The final excited state is a metastable state: the lowest $d$ electronic manifold of the $\mathrm{Ce}^{3+}$ from which the luminescence occurs. Therefore, when the negative (with respect to the lattice) $\mathrm{Mg}^{2+}$ ions are incorporated, it is likely that they shorten the $\mathrm{Ce}^{3+}$ luminescence rise time by shortening the free holes lifetime. Following the above consideration one can distinguish tree contributions to the luminescence rise time observer in the YAG:Ce and YAG:Ce,Mg systems. They are the free electron-hole pair lifetime (thermalization time and thermalized electron-hole pairs related to the capturing of $\mathrm{Ce}^{3+}$ or $\mathrm{Ce}^{3+}, \mathrm{Mg}^{2+}$ ) and the intra-configurational relaxation time in $\mathrm{Ce}^{3+}$ ion. Since the total lifetime is very short in the case of YAG:Ce, $\mathrm{Mg}$, the intra-configurational relaxation time and the electron-hole pair termalization lifetime also have to be very short. Thus as far as the YAG:Ce luminescence rise time is considered, although our calculations do not yield the unambiguous values of lifetimes in particular excited states, the total lifetime of $100 \mathrm{ps}$ should be related mainly to the thermalized electron-hole pairs.

\section{Acknowledgments}

The work presented in this paper was supported by the Committee for Scientific Research grant 2P03B003 13 and the University of Gdansk research grant BW 5200-5-0192-8. We wish to thank DAAD for granting MCP used as a detector of single photons.

\section{References}

[1] D.S. Hamilton, S.K. Gayen, G.J. Pogatshnik, R.D. Ghen, W.J. Miniscalco, Phys. Rev. B 39, 8807 (1988).

[2] D.S. Hamilton, in: Proc. First Intern. Conf. Tunable Solid State Lasers, La Jolla (California) 1984, Eds. P. Hammerling, A.B Budgov, A. Pinto, Springer-Verlag, Berlin 1985, p. 80.

[3] E. Zych, C. Brecher, A.J. Wojtowicz, H. Lingertat, J. Lumin. 75, 193 (1997). 
[4] Li-Ji Lyu, D.S. Hamilton, J. Lumin. 48\&49, 251 (1991).

[5] K. Wiśniewski, Cz. Koepke, A.J. Wojtowicz, W. Drozdowski, M. Grinberg, S. Kaczmarek, J. Kisielewski, Acta Phys. Pol. A 95, 403 (1999). 